\title{
Administered Activities in Pediatric Nuclear Medicine and the Impact of the 2010 North American Consensus Guidelines on General Hospitals in the United States
}

\author{
Frederic H. Fahey ${ }^{1}$, Sonja I. Ziniel ${ }^{2-4}$, Dacie Manion ${ }^{5}$, Amanda Baker ${ }^{1}$, and S. Ted Treves ${ }^{1,6}$ \\ ${ }^{I}$ Department of Radiology, Boston Children's Hospital, Harvard Medical School, Boston, Massachusetts; ${ }^{2}$ Program for Patient Safety \\ and Quality, Center for Patient Safety and Quality Research, Boston Children's Hospital, Harvard Medical School, Boston, \\ Massachusetts; ${ }^{3}$ Division of Adolescent and Young Adult Medicine, Boston Children's Hospital, Harvard Medical School, Boston, \\ Massachusetts; ${ }^{4}$ Department of Pediatrics, Harvard Medical School, Boston, Massachusetts; ${ }^{5}$ Department of Mechanical \\ Engineering, Massachusetts Institute of Technology, Cambridge, Massachusetts; and ${ }^{6}$ Department of Radiology, Brigham and \\ Women's Hospital, Harvard Medical School, Boston, Massachusetts
}

We sought to describe the practice of pediatric nuclear medicine at general hospitals in the United States and to assess the impact of dose awareness campaigns such as Image Gently. Methods: A web-based survey was developed that requested information regarding hospital type, whether the hospital practices pediatric nuclear medicine, and the hospital's method for determining the administered activity for children. The survey invitation was emailed to a sample of general hospitals with more than 300 beds, excluding dedicated pediatric, veterans, psychiatric, and rehabilitation hospitals. Data were collected for 5 procedures performed on children: 99mTc-methylene diphosphate (MDP) bone scans, ${ }^{99 m T c-m e r c a p t o a c e t y l-~}$ triglycine (MAG3) renograms, $99 \mathrm{mTc}$-dimercaptosuccinic acid (DMSA) renal cortical scans, ${ }^{99 m T c}$-based hepatobiliary scans, and ${ }^{18} \mathrm{~F}-\mathrm{FDG}$ PET scans. The sites reported dosage by weight $(\mathrm{MBq} / \mathrm{kg})$, minimum and maximum dosages, and the activities that they would administer to 2 hypothetical patients: a 5 -y-old boy (20 kg, $110 \mathrm{~cm}$ tall) and a 10-y-old girl $(30 \mathrm{~kg}, 140 \mathrm{~cm}$ tall). Results: The invitation was delivered to 196 sites, with 121 $(61.7 \%)$ responding. Eighty-two hospitals (67.8\%) performed nuclear medicine on children. All sites scaled administered activity for children, mostly by body weight. Also, $82.4 \%$ of sites indicated they were familiar with Image Gently, $57.1 \%$ were familiar with the 2010 North American consensus guidelines for children, and $54.9 \%$ altered their protocols because of the guidelines. The median value for parameters defined by the guidelines was equal to the guideline-recommended value for all procedures. More than $50 \%$ of the sites-particularly those familiar with the guidelineswere compliant with the guidelines regarding both the acquisition parameters and the administered activities for the 2 hypothetical patients. However, there remained a wide variation in practice, sometimes by more than a factor of 10 , for sites not familiar with the guidelines. Conclusion: Image Gently and the North American guidelines have had a substantial impact on pediatric nuclear medicine practice in the United States. However, a wide variation in practice still exists, particularly for sites not familiar with the guidelines. Further promotion and dissemination of the guidelines and best practice are still necessary.

Received Jan. 5, 2016; revision accepted Mar. 8, 2016

For correspondence or reprints contact: Frederic H. Fahey, Division of Nuclear Medicine, Boston Children's Hospital, 300 Longwood Ave., Boston, MA 02115.

E-mail: frederic.fahey@childrens.harvard.edu

Published online Apr. 7, 2016.

COPYRIGHT (C 2016 by the Society of Nuclear Medicine and Molecular Imaging, Inc.
Key Words: pediatric nuclear medicine; radiopharmaceutical dosing; North American guidelines; Image Gently

J Nucl Med 2016; 57:1478-1485

DOI: 10.2967/jnumed.116.172148

$\mathbf{T}$

he clinical value of pediatric nuclear medicine is well established, providing essential information to the clinician across many medical specialties, including urology, neurology, oncology, and orthopedics $(1,2)$. However, administration of radiopharmaceuticals exposes the patient to ionizing radiation. Although there is no direct evidence that the radiation doses encountered in nuclear medicine lead to adverse health effects, a report from the U.S. National Academy of Sciences supports the concept that there is a small potential risk even for very low doses (3). The report also states that the risk for radiation-induced cancer may be higher in children than in adults, perhaps by a factor of 2 or 3 , although this likely varies by tumor type, age, and sex (4). Therefore, it is prudent to optimize administered activity for pediatric nuclear medicine as a function of body size, maintaining the dose to the patient as low as possible while still providing essential clinical information $(5,6)$.

In 2008, we reported on a survey of premiere North American pediatric institutions that demonstrated a wide variation in the administered activities for children (7). Because of these results and a general interest in radiation dose optimization as exemplified by the Image Gently campaign (8), the 2010 North American consensus guidelines for administered activity in children and adolescents were developed by an expert working group convened by the American College of Radiology, the Society of Pediatric Radiology, and the Society of Nuclear Medicine and Molecular Imaging (9). These guidelines were subsequently publicized through publication in several journals, including The Journal of Nuclear Medicine; presentations at national meetings; notifications on the websites of the Image Gently campaign and the Society of Nuclear Medicine and Molecular Imaging; and delivery of a "Go with the Guidelines" poster to every nuclear medicine clinic in North America. A follow-up survey of the same institutions in 2013 showed that the sites had generally reduced their administered 
activities in children, particularly for those procedures covered by the guidelines. Variability in the administered activities was also reduced (10).

Because most children in the United States are not treated at dedicated pediatric hospitals, pediatric nuclear medicine is practiced predominantly at general hospitals. Nuclear medicine practitioners at these institutions may not be as familiar with the guidelines as are practitioners at pediatric hospitals. Therefore, we sought to assess pediatric nuclear medicine practice within large general hospitals in the United States and their familiarity and compliance with the guidelines.

\section{MATERIALS AND METHODS}

This study was exempt from needing approval by the Institutional Review Board at Boston Children's Hospital because the survey asked the sites about their practice rather than about individual patients. The survey was completed in the spring of 2013.

\section{Questionnaire}

The questionnaire (available as supplemental material at http://jnm. snmjournals.org) included a background section regarding site characteristics, a practice section on the approach to adjusting the administered activities in children, and a section assessing familiarity with Image Gently and the guidelines and whether this familiarity altered the site's pediatric nuclear medicine practice. We inquired about hospital location (urban, suburban, or rural) and type (community, teaching, or university-based). We asked about the number of nuclear medicine procedures (in children and adults) performed annually and the number of nuclear medicine technologists and physicians performing and interpreting the studies, respectively.

The survey inquired about 5 procedures commonly performed on children: ${ }^{99 \mathrm{~m}} \mathrm{Tc}-\mathrm{methylene}$ diphosphate (MDP) bone scans, ${ }^{99 \mathrm{~m}} \mathrm{Tc}-$ mercaptoacetyltriglycine (MAG3) renal scans, ${ }^{99} \mathrm{~m}$ Tc-dimercaptosuccinic acid (DMSA) renal scans, ${ }^{99 \mathrm{~m}} \mathrm{Tc}$-iminodiacetic acid derivative (HIDA) hepatobiliary scans, and ${ }^{18}$ F-FDG PET. We asked whether the hospitals performed these procedures on children, whether they scaled the administered activity according to the child's size, and, if so, how this scaling was applied. The sites provided their administered activity per body weight $(\mathrm{MBq} / \mathrm{kg})$ as well as the minimum and the maximum activities for smaller and larger patients, respectively. We inquired about the amount of activity they would administer for 2 hypothetical patients, a 5-y-old boy weighing $20 \mathrm{~kg}$ and $110 \mathrm{~cm}$ tall and a $10-\mathrm{y}$-old girl weighing $30 \mathrm{~kg}$ and $140 \mathrm{~cm}$ tall.

The sites were asked whether they were familiar with Image Gently and the 2010 guidelines or "Go with the Guidelines" campaign. Finally, they were asked whether they had altered any of their protocols for children because of the guidelines. The questionnaire was tested in 10 hospitals across the United States through cognitive interviews, which indicated that it could be completed in less than $15 \mathrm{~min}$. The test hospitals were excluded from the eligible list of hospitals. Invitations for the web-based survey were emailed in March 2013 to the nuclear medicine chief technologist or supervisor at each site. Two reminders were sent, and the survey was closed after $30 \mathrm{~d}$.

\section{Hospital Sample}

Hospitals with over 300 beds were selected from the 2010 American Hospital Association database (11) since these were likely to maintain a nuclear medicine service. Sites were excluded if they were pediatric, veterans, psychiatric, or rehabilitation facilities. From the 806 eligible hospitals, 270 were selected, as this provided an adequately sized sample using essentially random sampling while ensuring wide geographic distribution across the United States. To maximize compliance and reliability, we attempted to contact each site by phone to obtain the email address of the nuclear medicine chief technologist or supervisor. Up to 5 contact attempts were made to each site.

\section{Statistical Methods}

The characteristics of the hospitals and nuclear medicine services, as well as their use of the 5 imaging procedures, are presented using descriptive statistics (percentages, means, SDs, and medians). A "factor of variation," used in our previous surveys, was calculated for each parameter by dividing the highest (maximum) value reported by one site by the lowest (minimum) value reported by another $(7,10)$. For parameters listed in the guidelines and the administered activities for the 2 hypothetical patients, we tabulated the percentage of respondents who were within $20 \%$ of the guideline. We considered a $20 \%$ variance to represent basic compliance because this is the variance allowed by the U.S. Nuclear Regulatory Commission for the administered activity for a particular patient relative to the prescribed value (12). The administered activities for the 2 hypothetical patients were displayed in box plots indicating the 10th and 90th percentiles, the 25th and 75th percentiles, the median, and the administered activity recommended by the guidelines.

\section{RESULTS}

The email address of the nuclear medicine chief technologist or supervisor was obtained for 208 of the 270 sites, and the invitation was emailed to these 208 sites (Fig. 1). Twelve emails were returned as not deliverable, and thus 196 sites received the invitation. Of these 196 sites, 121 completed and returned the survey forms $(61.7 \%)$.

Eighty-two of the 121 sites $(67.8 \%)$ indicated that they perform nuclear medicine studies on children. Descriptive statistics of the sites are presented in Table 1 . The 82 sites were split evenly between urban and suburban settings ( $49.4 \%$ and $48.2 \%$, respectively), with

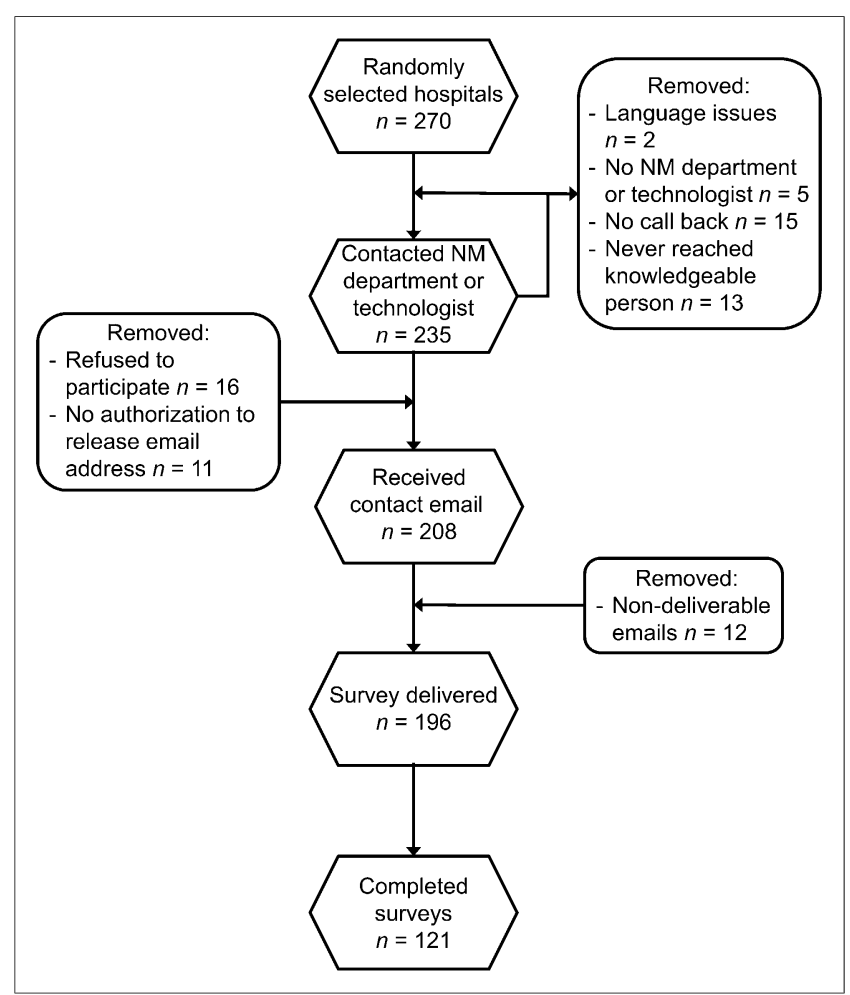

FIGURE 1. Flowchart illustrating sampling of hospitals and survey response rate. $\mathrm{NM}=$ nuclear medicine. 
TABLE 1

Characteristics of All 121 Sites and the Subset of 82 Sites Performing Pediatric Nuclear Medicine

\begin{tabular}{|c|c|c|c|c|}
\hline \multirow[b]{2}{*}{ Characteristic } & \multicolumn{2}{|c|}{ All 121} & \multicolumn{2}{|c|}{ Subset of 82} \\
\hline & $n$ & Data & $n$ & Data \\
\hline \multicolumn{5}{|l|}{ Location of hospital } \\
\hline Total & 119 & & 81 & \\
\hline Rural (population, $<30,000$ ) & 3 & $2.5 \%$ & 2 & $2.5 \%$ \\
\hline Suburban (population, $30,000-500,000$ ) & 57 & $47.9 \%$ & 39 & $48.2 \%$ \\
\hline Urban (population, $>500,000$ ) & 59 & $49.6 \%$ & 40 & $49.4 \%$ \\
\hline \multicolumn{5}{|l|}{ Hospital type } \\
\hline Total & 121 & & 82 & \\
\hline Community hospital, nonteaching & 27 & $22.3 \%$ & 12 & $14.6 \%$ \\
\hline Community hospital, teaching & 62 & $51.3 \%$ & 45 & $54.9 \%$ \\
\hline Large academic hospital & 31 & $25.6 \%$ & 24 & $29.3 \%$ \\
\hline Government-affiliated hospital & 1 & $0.8 \%$ & 1 & $1.2 \%$ \\
\hline \multicolumn{5}{|l|}{ Affiliated with medical school? } \\
\hline Total & 120 & & 82 & \\
\hline No & 58 & $48.3 \%$ & 38 & $46.3 \%$ \\
\hline Yes & 62 & $51.7 \%$ & 44 & $53.7 \%$ \\
\hline \multicolumn{5}{|l|}{ Residents in hospital? } \\
\hline Total & 116 & & 81 & \\
\hline No & 30 & $25.9 \%$ & 17 & $21.0 \%$ \\
\hline Yes & 86 & $74.1 \%$ & 64 & $79.0 \%$ \\
\hline \multicolumn{5}{|l|}{ Medical students in hospital? } \\
\hline Total & 115 & & 81 & \\
\hline No & 28 & $24.4 \%$ & 17 & $21.0 \%$ \\
\hline Yes & 87 & $76.6 \%$ & 64 & $79.0 \%$ \\
\hline \multicolumn{5}{|c|}{ No. of physicians in nuclear medicine department } \\
\hline Total & 121 & $7.1(11.46)$ & 82 & $7.9(10.87)$ \\
\hline \multicolumn{5}{|c|}{ No. of nuclear technologists in nuclear medicine department } \\
\hline Total & 101 & $8.0(5.73)$ & 79 & $8.4(5.86)$ \\
\hline \multicolumn{5}{|l|}{ No. of nuclear medicine studies annually } \\
\hline Total & 101 & & 79 & \\
\hline$<3,000$ & 21 & $20.8 \%$ & 14 & $17.7 \%$ \\
\hline $3,000-6,000$ & 55 & $54.5 \%$ & 45 & $57.0 \%$ \\
\hline 6,000 & 25 & $24.8 \%$ & 20 & $25.3 \%$ \\
\hline \multicolumn{5}{|c|}{ Nuclear medicine studies on children performed at hospital? } \\
\hline Total & 104 & & & \\
\hline No & 21 & $20.2 \%$ & - & - \\
\hline Yes & 83 & $79.8 \%$ & - & - \\
\hline \multicolumn{5}{|c|}{ No. of nuclear medicine studies on children annually } \\
\hline Total & 72 & $189.0(389.39)$ & 71 & $191.5(391.57)$ \\
\hline \multicolumn{5}{|c|}{ Familiarity with doses recommended by 2010 guidelines? } \\
\hline Total & & & 49 & \\
\hline No & - & - & 21 & $42.9 \%$ \\
\hline Yes & - & - & 28 & $57.1 \%$ \\
\hline \multicolumn{5}{|c|}{ Familiarity with Image Gently or "Go with the Guidelines"? } \\
\hline Total & & & 51 & \\
\hline No & - & - & 9 & $17.7 \%$ \\
\hline Yes & - & - & 42 & $82.3 \%$ \\
\hline \multicolumn{5}{|c|}{ Any imaging protocol altered because of guidelines? } \\
\hline Total & & & 51 & \\
\hline No & - & - & 23 & $45.1 \%$ \\
\hline Yes & - & - & 28 & $54.9 \%$ \\
\hline
\end{tabular}

1480 The Journal of Nuclear Medicine • Vol. 57 • No. 9 • September 2016 
TABLE 2

Use and Patterns of Use for Radiopharmaceutical Agents in 5 Commonly Performed Pediatric Procedures

\begin{tabular}{lc}
\hline \multicolumn{1}{c}{ Agent } & Percentage of the 82 sites \\
\hline Single & \\
\hline 99mTc-MDP & $86.6 \%$ \\
99mTc-MAG3 & $68.3 \%$ \\
99mTc-DMSA & $59.8 \%$ \\
99mTc-HIDAs & $39.0 \%$ \\
18F-FDG & $30.5 \%$ \\
Combination & \\
All 5 & $16.9 \%$ \\
4 of 5 & $23.4 \%$ \\
3 of 5 & $19.5 \%$ \\
2 or less of 5 & $40.3 \%$ \\
\hline
\end{tabular}

only $2.5 \%$ of sites being in rural settings. Most sites were community teaching hospitals (54.9\%); $29.3 \%$ and $14.6 \%$ were described as large academic and community nonteaching hospitals, respectively.
Most sites (82.3\%) performed more than 3,000 nuclear medicine procedures a year. The number of pediatric nuclear medicine procedures varied widely (median, 40; mean $\pm \mathrm{SD}, 191.5 \pm 391.6$ ). Most sites $(53.5 \%)$ performed under 50 pediatric procedures per year, whereas less than $10 \%$ performed 500 or more per year. Of those performing pediatric nuclear medicine, $82.4 \%$ were familiar with Image Gently, $57.1 \%$ were familiar with the 2010 guidelines or the "Go with the Guidelines" poster, and 54.9\% indicated that they had altered their protocols because of the guidelines. These percentages did not vary with respect to hospital type; that is, the percentages were essentially the same for community nonteaching hospitals and large academic hospitals. Of those performing pediatric nuclear medicine, $86.6 \%$ performed ${ }^{99 \mathrm{~m}} \mathrm{Tc}-\mathrm{MDP}$ bone scans, 68.3\% 99m Tc-MAG3 renograms, 59.8\% 99m Tc-DMSA scans, $39.0 \%$ hepatobiliary studies using $99 \mathrm{~m}$ Tc-HIDA derivatives, and $30.5 \%{ }^{18} \mathrm{~F}-\mathrm{FDG}$ whole-body PET studies (Table 2). All 5 types of studies were performed at $16.9 \%$ of the sites, whereas $40.3 \%$ performed only 1 or 2 types.

The results of the survey are summarized in Tables 3-5. For ${ }^{99 m}$ Tc-MAG3, the guidelines recommend 2 values, a higher value if an initial radionuclide angiogram is acquired (flow phase) and a lower value if no flow phase is acquired. The guideline-recommended ${ }^{99 m}$ Tc-MAG3 value for a flow-phase acquisition was used for comparison in Tables 3-5 and Figure 2. The lower compliance

TABLE 3

Radiopharmaceutical Dosage per Kilogram of Body Weight, According to Familiarity of Site with 2010 Guidelines

\begin{tabular}{|c|c|c|c|c|c|c|c|c|c|}
\hline \multirow{2}{*}{$\begin{array}{l}\text { Familiar with } \\
\text { guidelines? }\end{array}$} & \multirow[b]{2}{*}{$n$} & \multicolumn{5}{|c|}{ Dosage* } & \multirow{2}{*}{$\begin{array}{c}\text { Factor of } \\
\text { variation } \\
\text { (max/min) }\end{array}$} & \multirow{2}{*}{$\begin{array}{l}\text { Guideline- } \\
\text { recommended } \\
\text { dosage }^{\star}\end{array}$} & \multirow{2}{*}{$\begin{array}{c}\text { Respondents } \\
\text { following } \\
\text { guideline } \pm 20 \%\end{array}$} \\
\hline & & Minimum & Mean & $\mathrm{SD}$ & Median & Maximum & & & \\
\hline \multicolumn{10}{|l|}{ 99mTc-MDP } \\
\hline Total & 39 & $5.18(0.14)$ & $11.08(0.30)$ & $7.32(0.20)$ & $9.25(0.25)$ & $41.63(1.13)$ & 8.04 & $9.25(0.25)$ & $84.6 \%$ \\
\hline No & 10 & $5.18(0.14)$ & $12.75(0.34)$ & $10.30(0.28)$ & $10.36(0.28)$ & $41.63(1.13)$ & 8.04 & & $80.0 \%$ \\
\hline Yes & 24 & $6.29(0.17)$ & $9.02(0.24)$ & $0.82(0.02)$ & $9.25(0.25)$ & $10.36(0.28)$ & 1.65 & & $95.8 \%$ \\
\hline \multicolumn{10}{|l|}{ 99mTc-MAG3 } \\
\hline Total & 34 & $2.59(0.07)$ & $6.33(0.17)$ & $6.73(0.18)$ & $5.55(0.15)$ & $41.63(1.13)$ & 16.07 & $5.55(0.15)$ & $58.8 \%$ \\
\hline No & 8 & $2.59(0.07)$ & $10.85(0.29)$ & $13.41(0.36)$ & $5.18(0.14)$ & $41.63(1.13)$ & 16.07 & & $50.0 \%$ \\
\hline Yes & 24 & $3.70(0.10)$ & $4.83(0.13)$ & $0.90(0.02)$ & $5.55(0.15)$ & $5.55(0.15)$ & 1.50 & & $62.5 \%$ \\
\hline \multicolumn{10}{|l|}{${ }^{99 m}$ Tc-DMSA } \\
\hline Total & 28 & $1.11(0.03)$ & $2.02(0.05)$ & $0.56(0.02)$ & $1.85(0.05)$ & $3.70(0.10)$ & 3.33 & $1.85(0.05)$ & $78.6 \%$ \\
\hline No & 6 & $1.85(0.05)$ & $2.41(0.07)$ & $0.73(0.02)$ & $2.22(0.06)$ & $3.70(0.10)$ & 2.00 & & $50.0 \%$ \\
\hline Yes & 21 & $1.11(0.03)$ & $1.92(0.05)$ & $0.48(0.01)$ & $1.85(0.05)$ & $3.70(0.10)$ & 3.33 & & $85.7 \%$ \\
\hline \multicolumn{10}{|l|}{ 99mTc-HIDAs } \\
\hline Total & 21 & $1.48(0.04)$ & $4.14(0.11)$ & $4.62(0.12)$ & $1.85(0.05)$ & $18.50(0.50)$ & 12.50 & $1.85(0.05)$ & $61.9 \%$ \\
\hline No & 6 & $1.85(0.05)$ & $6.00(0.16)$ & $4.96(0.13)$ & $3.93(0.11)$ & $14.80(0.40)$ & 8.00 & & $16.7 \%$ \\
\hline Yes & 14 & $1.48(0.04)$ & $3.54(0.10)$ & $4.57(0.12)$ & $1.85(0.05)$ & $18.50(0.50)$ & 12.50 & & $78.6 \%$ \\
\hline \multicolumn{10}{|l|}{${ }^{18} \mathrm{~F}-\mathrm{FDG}$} \\
\hline Total & 18 & $2.22(0.06)$ & $4.97(0.13)$ & $1.87(0.05)$ & $4.44(0.12)$ & $10.58(0.29)$ & 4.77 & $3.7-5.18(0.10-0.14)$ & $77.8 \%$ \\
\hline No & 3 & $3.70(0.10)$ & $4.81(0.13)$ & $0.98(0.03)$ & $5.18(0.14)$ & $5.55(0.15)$ & 1.50 & & $100.0 \%$ \\
\hline Yes & 13 & $2.22(0.06)$ & $4.35(0.12)$ & $1.00(0.03)$ & $4.44(0.12)$ & $6.29(0.17)$ & 2.83 & & $84.6 \%$ \\
\hline
\end{tabular}

*Units of measurement are megabecquerels per kilogram $(\mathrm{MBq} / \mathrm{kg})$ followed by millicuries per kilogram $(\mathrm{mCi} / \mathrm{kg})$ in parentheses.

$n$ indicates number of respondents. In some cases, numbers answering "no" and "yes" do not equal total number because not all sites indicated whether they are familiar with 2010 guidelines. 
TABLE 4

Minimum and Maximum Radiopharmaceutical Dosages in Smaller and Larger Patients, Respectively, According to Familiarity of Site with 2010 Guidelines

\begin{tabular}{|c|c|c|c|c|c|c|c|c|c|}
\hline \multirow{2}{*}{$\begin{array}{l}\text { Familiar with } \\
\text { guidelines? }\end{array}$} & \multirow[b]{2}{*}{$n$} & \multicolumn{5}{|c|}{ Dosage $^{*}$} & \multirow{2}{*}{$\begin{array}{l}\text { Factor of variation } \\
\text { (max/min) }\end{array}$} & \multirow{2}{*}{$\begin{array}{l}\text { Guideline- } \\
\text { recommended } \\
\text { dosage }^{\star}\end{array}$} & \multirow{2}{*}{$\begin{array}{c}\text { Respondents } \\
\text { following } \\
\text { guideline } \pm 20 \%\end{array}$} \\
\hline & & Minimum & Mean & SD & Median & Maximum & & & \\
\hline \multicolumn{10}{|c|}{$\begin{array}{l}\text { Minimum dose } \\
\text { in smaller patients }\end{array}$} \\
\hline \multicolumn{10}{|l|}{ 99mTc-MDP } \\
\hline Total & 35 & $18.50(0.50)$ & 51.27 (1.39) & $35.07(0.95)$ & $37.00(1.00)$ & $185.00(5.00)$ & 10.00 & $37.00(1.00)$ & $71.4 \%$ \\
\hline No & 9 & $37.00(1.00)$ & 71.94 (1.94) & $39.73(1.07)$ & $74.00(2.00)$ & $148.00(4.00)$ & 4.00 & & $44.4 \%$ \\
\hline Yes & 21 & $18.50(0.50)$ & $39.64(1.07)$ & $12.11(0.33)$ & $37.00(1.00)$ & $74.00(2.00)$ & 4.00 & & $85.7 \%$ \\
\hline \multicolumn{10}{|l|}{ 99mTc-MAG3 } \\
\hline Total & 39 & $18.50(0.50)$ & 37.47 (1.01) & $15.00(0.41)$ & $37.00(1.00)$ & $111.00(3.00)$ & 6.00 & - & - \\
\hline No & 9 & $18.50(0.50)$ & $32.89(0.89)$ & $8.16(0.22)$ & $37.00(1.00)$ & $37.00(1.00)$ & 2.00 & & \\
\hline Yes & 26 & $18.50(0.50)$ & 37.71 (1.02) & $16.11(0.44)$ & $37.00(1.00)$ & $111.00(3.00)$ & 6.00 & & \\
\hline \multicolumn{10}{|l|}{${ }^{99 m}$ Tc-DMSA } \\
\hline Total & 33 & $18.50(0.50)$ & $32.07(0.87)$ & $26.63(0.72)$ & $18.50(0.50)$ & $111.00(3.00)$ & 6.00 & $18.5(0.50)$ & $63.6 \%$ \\
\hline No & 10 & $18.50(0.50)$ & $35.15(0.95)$ & $28.19(0.76)$ & $27.75(0.75)$ & $111.00(3.00)$ & 6.00 & & $50.0 \%$ \\
\hline Yes & 22 & $18.50(0.50)$ & $30.44(0.82)$ & $27.04(0.73)$ & $18.50(0.50)$ & $111.00(3.00)$ & 6.00 & & $72.7 \%$ \\
\hline \multicolumn{10}{|l|}{ 99mTc-HIDAs } \\
\hline Total & 22 & $18.50(0.50)$ & $28.59(0.77)$ & $13.66(0.37)$ & $18.50(0.50)$ & $74.00(2.00)$ & 4.00 & $18.5(0.50)$ & $54.6 \%$ \\
\hline No & 7 & $18.50(0.50)$ & $29.07(0.79)$ & $9.89(0.27)$ & $37.00(1.00)$ & $37.00(1.00)$ & 2.00 & & $42.9 \%$ \\
\hline Yes & 14 & $18.50(0.50)$ & $27.75(0.75)$ & $15.81(0.43)$ & $18.50(0.50)$ & $74.00(2.00)$ & 4.00 & & $64.3 \%$ \\
\hline \multicolumn{10}{|l|}{${ }^{18} \mathrm{~F}-\mathrm{FDG}$} \\
\hline Total & 16 & $18.50(0.50)$ & 63.59 (1.72) & 72.75 (1.97) & $37.00(1.00)$ & $296.00(8.00)$ & 16.00 & $37.00(1.00)$ & $75.0 \%$ \\
\hline No & 2 & $37.00(1.00)$ & $166.5(4.50)$ & $183.14(4.95)$ & $166.50(4.50)$ & $296.00(8.00)$ & 8.00 & & $50.0 \%$ \\
\hline Yes & 13 & $18.50(0.50)$ & $49.81(1.35)$ & $42.31(1.14)$ & $37.00(1.00)$ & $185.00(5.00)$ & 10.00 & & $76.9 \%$ \\
\hline \multicolumn{10}{|l|}{$\begin{array}{l}\text { Maximum dose in } \\
\text { larger patients }\end{array}$} \\
\hline \multicolumn{10}{|l|}{${ }^{99 m}$ Tc-MDP } \\
\hline Total & 50 & $444.00(12.00)$ & $788.92(21.32)$ & $152.01(4.11)$ & $740.00(20.00)$ & $1110.00(30.00)$ & 2.50 & - & - \\
\hline No & 16 & $444.00(12.00)$ & 797.81 (21.56) & 136.43 (3.69) & $740.00(20.00)$ & $925.00(25.00)$ & 2.08 & & \\
\hline Yes & 26 & $444.00(12.00)$ & $770.04(20.81)$ & $172.96(4.67)$ & $740.00(20.00)$ & $1,110.00(30.00)$ & 2.50 & & \\
\hline \multicolumn{10}{|l|}{ 99mTc-MAG3 } \\
\hline Total & 42 & $111.00(3.00)$ & $266.05(7.19)$ & $115.64(3.13)$ & $240.50(6.50)$ & $555.00(15.00)$ & 5.00 & - & - \\
\hline No & 11 & $148.00(4.00)$ & 299.36 (8.09) & $99.91(2.70)$ & $370.00(10.00)$ & $370.00(10.00)$ & 2.50 & & \\
\hline Yes & 27 & $111.00(3.00)$ & 246.67 (6.67) & 119.23 (3.22) & $185.00(5.00)$ & $555.00(15.00)$ & 5.00 & & \\
\hline \multicolumn{10}{|l|}{ 99mTc-DMSA } \\
\hline Total & 35 & $74.00(2.00)$ & $178.68(4.83)$ & $54.74(1.48)$ & $185.00(5.00)$ & $370.00(10.00)$ & 5.00 & - & - \\
\hline No & 11 & $74.00(2.00)$ & $164.82(4.45)$ & $44.90(1.21)$ & $185.00(5.00)$ & $222.00(6.00)$ & 3.00 & & \\
\hline Yes & 22 & $74.00(2.00)$ & $183.35(4.96)$ & $60.63(1.64)$ & $185.00(5.00)$ & $370.00(10.00)$ & 5.00 & & \\
\hline \multicolumn{10}{|l|}{ 99mTc-HIDAs } \\
\hline Total & 26 & $111.00(3.00)$ & $186.45(5.04)$ & $43.40(1.17)$ & $185.00(5.00)$ & $296.00(8.00)$ & 2.67 & - & - \\
\hline No & 8 & $148.00(4.00)$ & $194.25(5.25)$ & $43.10(1.16)$ & $185.00(5.00)$ & $296.00(8.00)$ & 2.00 & & \\
\hline Yes & 15 & $129.50(3.50)$ & $180.12(4.87)$ & $33.06(0.89)$ & $185.00(5.00)$ & $259.00(7.00)$ & 2.00 & & \\
\hline \multicolumn{10}{|l|}{${ }^{18} \mathrm{~F}-\mathrm{FDG}$} \\
\hline Total & 20 & $185.00(5.00)$ & $407.74(11.02)$ & $137.33(3.71)$ & $370.00(10.00)$ & $666.00(18.00)$ & 3.60 & - & - \\
\hline No & 5 & $370.00(10.00)$ & $458.80(12.40)$ & $92.87(2.51)$ & $444.00(12.00)$ & $555.00(15.00)$ & 1.50 & & \\
\hline Yes & 14 & $185.00(5.00)$ & $378.99(10.24)$ & $146.11(3.95)$ & $370.00(10.00)$ & $666.00(18.00)$ & 3.60 & & \\
\hline
\end{tabular}

*Units of measurement are megabecquerels $(\mathrm{MBq})$ followed by millicuries $(\mathrm{mCi})$ in parentheses.

$n$ indicates number of respondents. In some cases, numbers answering "no" and "yes" do not equal total number because not all sites indicated whether they are familiar with 2010 guidelines.

1482 The Journal of Nuclear Medicine • Vol. 57 • No. 9 • September 2016 
TABLE 5

Reported Dosages for the 2 Hypothetical Patients According to Familiarity of Site with 2010 Guidelines

\begin{tabular}{|c|c|c|c|c|c|c|c|}
\hline \multirow[b]{2}{*}{ Agent } & \multicolumn{3}{|c|}{ Respondents (n) } & \multicolumn{3}{|c|}{$\begin{array}{l}\text { Respondents following } \\
\text { guideline } \pm 20 \%\end{array}$} & \multirow[b]{2}{*}{ Guideline-recommended dosage } \\
\hline & Total & Yes & No & Total & Yes & No & \\
\hline \multicolumn{8}{|l|}{ Boy } \\
\hline 99mTc-MDP & 50 & 26 & 18 & $66.0 \%$ & $88.5 \%$ & $38.9 \%$ & $185.0(5)$ \\
\hline 99mTc-MAG3 & 42 & 27 & 12 & $45.2 \%$ & $55.6 \%$ & $25.0 \%$ & $111.0(3)$ \\
\hline 99mTc-DMSA & 37 & 23 & 12 & $54.1 \%$ & $69.6 \%$ & $25.0 \%$ & $37.0(1)$ \\
\hline 99mTc-HIDAs & 27 & 15 & 10 & $48.2 \%$ & $73.3 \%$ & $10.0 \%$ & $37.0(1)$ \\
\hline${ }^{18} \mathrm{~F}-\mathrm{FDG}$ & 20 & 14 & 5 & $70.0 \%$ & $78.6 \%$ & $60.0 \%$ & $74.0-103.6(2.0-2.8)$ \\
\hline \multicolumn{8}{|l|}{ Girl } \\
\hline 99mTc-MDP & 50 & 26 & 18 & $50.0 \%$ & $84.6 \%$ & $44.4 \%$ & $277.5(7.5)$ \\
\hline 99mTc-MAG3 & 43 & 27 & 12 & $45.2 \%$ & $55.6 \%$ & $25.0 \%$ & $166.5(4.5)$ \\
\hline 99mTc-DMSA & 37 & 23 & 12 & $51.4 \%$ & $65.2 \%$ & $33.3 \%$ & $55.5(1.5)$ \\
\hline 99mTc-HIDAs & 27 & 15 & 10 & $40.7 \%$ & $73.3 \%$ & $10.0 \%$ & $55.5(1.5)$ \\
\hline${ }^{18} \mathrm{~F}-\mathrm{FDG}$ & 20 & 14 & 5 & $70.0 \%$ & $85.7 \%$ & $60.0 \%$ & $111.0-155.4(3.0-4.2)$ \\
\hline
\end{tabular}

*Units of measurement are megabecquerels $(\mathrm{MBq})$ followed by millicuries $(\mathrm{mCi})$ in parentheses.

The hypothetical patients are a 5-y-old, $20-\mathrm{kg}, 110-\mathrm{cm}$ boy and a 10-y-old, 30-kg, 140-cm girl.

for this procedure may result from the fact that we did not inquire whether the site acquires a flow phase. Descriptive statistics, including the factor of variation, are tabulated in Tables 3 and 4 for each procedure and parameter (activity per body weight, minimum dose, and maximum dose). The results are listed for all respondents and are further categorized according to whether the site was familiar with the guidelines. For reference, the guideline-recommended values are listed. For those parameters addressed in the guidelines, the percentages of respondents that were within $20 \%$ of the guidelines are also reported. For all respondents and for those familiar with the guidelines, the median value exactly corresponded to the guidelines. More than $50 \%$ of the sites were within $20 \%$ of the guidelines for all parameters. For all respondents and for those familiar with the guidelines, more than $70 \%$ of the sites were within $20 \%$ of the guidelines for 5 of 9 and 7 of 9 parameters, respectively. In practically all cases, the median value of the parameter was lower for sites familiar with the guidelines than for those that were not.

Data for the 2 hypothetical patients are summarized in Table 5 and Figure 2. The median value coincided with the guideline recommendation in all cases, and except for ${ }^{99 m}$ Tc-MAG3, more than $48 \%$ of the respondents were compliant. The discrepancy for ${ }^{99 m} \mathrm{Tc}-\mathrm{MAG} 3$ may be due to the reasons stated previously. In general, compliance was considerably higher for sites familiar with the guidelines than for those that were not (Table 5).

\section{DISCUSSION}

In 2007, we performed a survey of dedicated pediatric hospitals in North America that indicated a substantial variability in pediatric nuclear medicine practice which, to a great extent, motivated the development of the 2010 guidelines. Our recently published follow-up survey of the same institutions indicated that the standardization of dosages resulting from the guidelines has had a significant impact on the practice of pediatric nuclear medicine. Because most children are treated at general and not pediatric hospitals, the question remained as to the variability of practice of pediatric nuclear medicine at these institutions. Also, we sought to assess the impact of Image Gently, the 2010 guidelines, and the "Go with the Guidelines" campaign on this practice.

We attribute the outstanding response rate of our survey (121 respondents to 196 invitations, $61.7 \%$ ) to the fact that we contacted all sites by telephone before the survey. Table 1 indicates that the subset of 82 sites performing studies on children had essentially the same characteristics as the complete set of 121 sites. Pediatric nuclear medicine is performed at a high percentage of general hospitals, although the number of pediatric studies varies significantly. We inquired specifically about 5 commonly performed pediatric nuclear medicine procedures. The largest number of sites performed ${ }^{99 \mathrm{~m}} \mathrm{Tc}-\mathrm{MDP}$ bone scans, with fewer sites performing ${ }^{99 \mathrm{~m} T c-M A G 3}$, ${ }^{99 \mathrm{~m} T c-D M S A}$, ${ }^{99 \mathrm{~m} T c-H I D A}$, and ${ }^{18} \mathrm{~F}$-FDG PET studies. Several sites performed only 1 or 2 of these procedures.

The Alliance for Radiation in Pediatric Imaging has, through its Image Gently campaign, sought to raise awareness regarding the appropriate use of pediatric imaging and the importance of radiation dose optimization. The Image Wisely campaign seeks the same in adult imaging. The impact of these campaigns in achieving these goals has remained uncertain. Eighty-three percent of the sites that performed studies on children were familiar with Image Gently, 57.1\% were familiar with the 2010 guidelines or the "Go with the Guidelines" campaign, and $54.9 \%$ indicated that they had altered their image protocols in children because of the guidelines. These percentages did not vary significantly with respect to hospital type-teaching or otherwise. Thus, a large percentage of hospital-based nuclear medicine services are familiar with Image Gently, more than half are familiar with the guidelines, and a very high percentage of those 
Boy, 5 y old, $20 \mathrm{~kg}, 110 \mathrm{~cm} \quad$ Girl, $10 \mathrm{y}$ old, $30 \mathrm{~kg}, 140 \mathrm{~cm}$
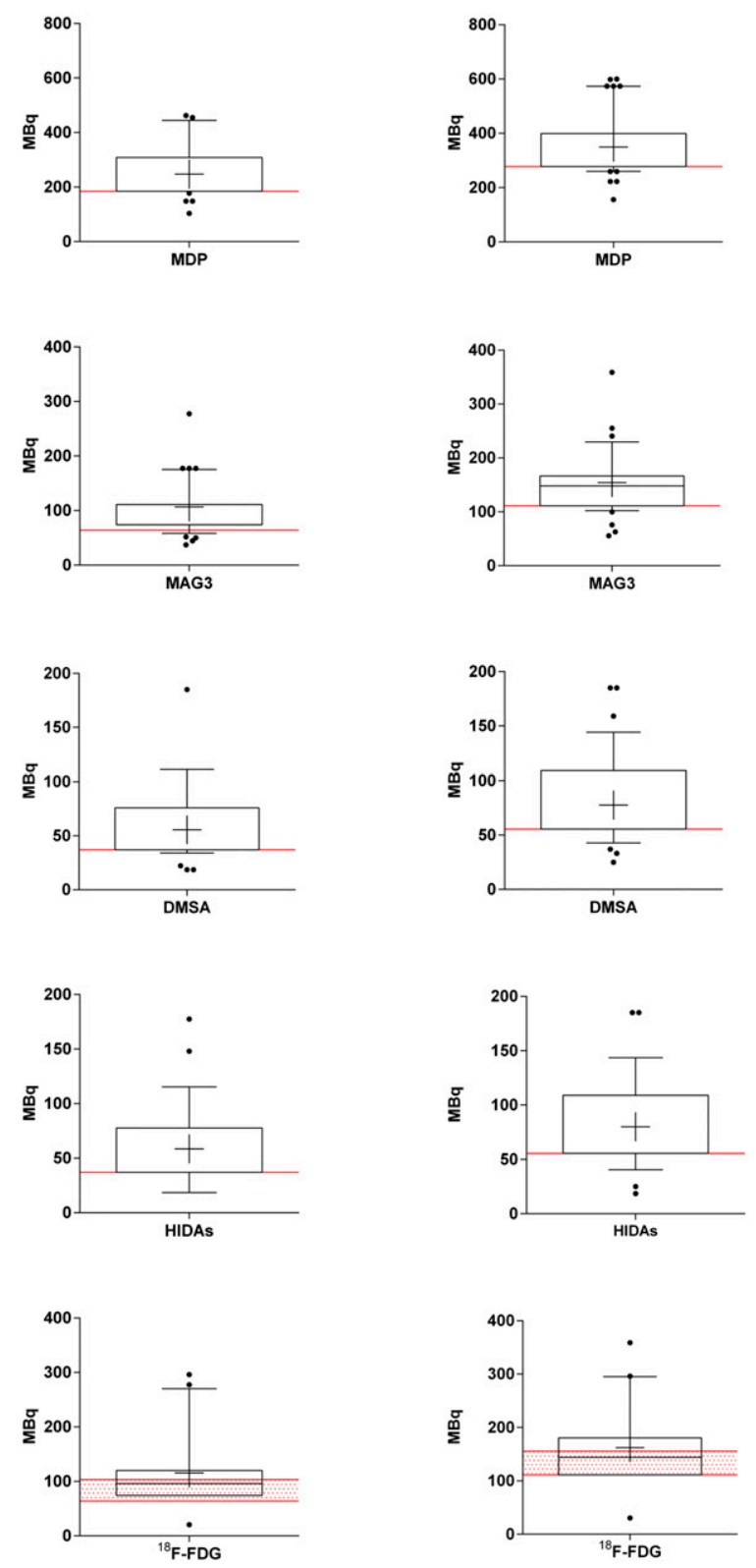

FIGURE 2. Box plots of administered activities for 2 hypothetical patients $(5$-y-old boy, $20 \mathrm{~kg}, 110 \mathrm{~cm}$ tall, and 10 -y-old girl, $30 \mathrm{~kg}$, $140 \mathrm{~cm}$ tall) for $99 \mathrm{mTc}-M D P$ bone scans (A and B), 99mTc-MAG3 renograms (C and D), ${ }^{99 m} T c-D M S A$ renal cortical scans ( $E$ and $\left.F\right)$, ${ }^{99 m} T c-$ HIDA derivative hepatobiliary scans $(G$ and $H)$, and ${ }^{18} \mathrm{~F}-\mathrm{FDG}$ torso PET scans (I and J). For each panel, median is shown by cross-hairs and tick to right of vertical axis, box represents 25 th and 75 th percentiles, whiskers represent 10th and 90th percentiles, and values outside whiskers are shown as dots. Guideline-recommended value for these patients is shown in red. Range of activities recommended for ${ }^{18} \mathrm{~F}-\mathrm{FDG}$ PET is shown by red hatching.

familiar with the guidelines altered their pediatric protocols because of the guidelines.

The median values of the reported administered activity parameters were consistent with the guidelines in all cases (Tables 3 and
4). Values substantially higher or lower than the recommendations may be of concern since too little activity may lead to an inadequate study and too much may lead to an unnecessary radiation dose. More than $58 \%$ of the sites were compliant with the guideline recommendations. In addition, sites familiar with the guidelines were substantially more compliant in all cases than those not familiar. For the 2 hypothetical patients, the median administered activity equaled the guideline recommendation and more than $40 \%$ of the sites and more than $65 \%$ of those familiar with the guidelines were compliant in all cases except for ${ }^{99}$ Tc-MAG3 (for which the discrepancy has been previously explained). We chose to include the ${ }^{99 m}$ Tc-MAG3 findings in this report because this procedure is very commonly performed on children (69\% of sites in this survey) and thus the results are of interest and informative.

Although a majority of the sites were compliant with the guidelines, a wide variation in the reported data still exists. Averaging across the 5 procedures, our factor of variation for the dosage per body weight was 8.94 , with a maximum of greater than 10 in some cases, indicating a rather wide variation in practice. However, this value was substantially reduced for sites familiar with the guidelines; the average factor of variation for such sites was 4.26. For the minimum dosage in smaller patients, the average factor of variation was 8.40 for all sites and 6.00 for sites familiar with the guidelines. For the maximum dosage in larger patients, the average factor of variation was 3.75 for all sites and 3.62 for sites familiar with the guidelines. The same is seen for the hypothetical patients (Fig. 2), which had a relatively narrow interquartile (25th to 75 th percentiles) range, generally less than a factor of 2. However, the full range of data (or even the 10th to 90th percentiles) demonstrates a substantially higher variation. It is possible that some of this variation was caused by inaccurate reporting, typographic errors, or a lack of understanding by some of the respondents of what was being asked. The consistency of response across several values in the outlying datasets argues against typographic errors, and the gathering of results across numerous sites provided suitable statistics, leading us to believe that most of this variation is likely real and representative of the practice of pediatric nuclear medicine.

This investigation demonstrates the substantial impact that Image Gently and the "Go with the Guidelines" campaign had on the practice of pediatric nuclear medicine in the United States. It also shows the wide variation in practice for those hospitals not familiar with the guidelines. Efforts remain ongoing by the Society of Nuclear Medicine and Molecular Imaging, the European Association of Nuclear Medicine (EANM), and the Image Gently campaign to bring attention to both the North American guidelines and the EANM pediatric dosage card and provide web-based tools to assist in their application $(13,14)$. More recently, the Nuclear Medicine Global Initiative has provided a list of helpful resources regarding pediatric nuclear medicine $(2,15)$.

This report has several limitations. There is a possibility that the respondents represent a biased sample, as those familiar with the guidelines may have been more likely to respond. However, the high response rate $(61.7 \%)$ reduces the possibility of such bias. In our attempt to keep the survey as concise as possible, we neglected to inquire about certain aspects of the procedures that may have been helpful to know, such as whether the ${ }^{99 m}$ Tc-MAG3 studies were acquired with or without a flow phase. It also may have been helpful to know the type of PET scanner each site used or the most likely indication for procedures being performed. Lastly, the survey was performed in 2013, before the publication of the 
2014 North American consensus guidelines that have been harmonized with the EANM pediatric dosage card (16). Therefore, a follow-up survey to assess the impact of the new guidelines may be warranted.

\section{CONCLUSION}

Most general hospitals in the United States perform nuclear medicine studies on children. Practically all hospitals scale the administered activity in children, with most simply scaling the adult dosage by body weight. Eighty-two percent of the sites indicated that they were familiar with Image Gently, $57.1 \%$ were familiar with the 2010 guidelines or the "Go with the Guidelines" campaign, and $54.9 \%$ of the sites indicated that they had altered their protocols for imaging children because of the guidelines. The median value for all reported parameters defined by the guidelines was equal to the guideline-recommended value. More than $50 \%$ of the sites were compliant with the guidelines with respect to both the acquisition parameters and the administered activities for the 2 hypothetical patients, and compliance was particularly high for sites familiar with the guidelines. However, there remains a wide variation in the reported data-by more than a factor of 10 in some cases-for sites not familiar with the guidelines. Further promotion and dissemination of the guidelines and best practice are still necessary.

\section{DISCLOSURE}

The costs of publication of this article were defrayed in part by the payment of page charges. Therefore, and solely to indicate this fact, this article is hereby marked "advertisement" in accordance with 18 USC section 1734 . No potential conflict of interest relevant to this article was reported.

\section{ACKNOWLEDGMENTS}

We would like to acknowledge Thien Nguyen for her assistance in contacting potential sites prior to the launching of the survey. We are also profoundly thankful to all sites that took the time to answer the survey and provide such a wealth of information.

\section{REFERENCES}

1. Treves ST. Pediatric Nuclear Medicine and Molecular Imaging. New York, NY: Springer; 2014.

2. Fahey FH, Bom HH-S, Chiti A, et al. Standardization of administered activities in pediatric nuclear medicine: a report of the first nuclear medicine global initiative project, part 1 -statement of the issue and a review of available resources. J Nucl Med. 2015;56:646-651.

3. Committee to Assess Health Risks from Exposure to Low Levels of Ionizing Radiation, National Research Council of the National Academies. Health Risks from Exposure to Low Levels of Ionizing Radiation: BEIR VII Phase 2. Washington, DC: The National Academies Press; 2006.

4. United Nations Scientific Committee on the Effects of Atomic Radiation. Sources, Effects and Risks of Ionizing Radiation: UNSCEAR 2013 Report. Vol II. Scientific Annex B: Effects of Radiation Exposure of Children. New York, NY: United Nations; 2013.

5. Fahey FH, Treves ST, Adelstein SJ. Minimizing and communicating radiation risk in pediatric nuclear medicine. J Nucl Med. 2011;52:1240-1251.

6. Treves ST, Falone AE, Fahey FH. Pediatric nuclear medicine and radiation dose. Semin Nucl Med. 2014;44:202-209.

7. Treves ST, Davis RT, Fahey FH. Administered radiopharmaceutical doses in children: a survey of 13 pediatric hospitals in North America. J Nucl Med. 2008;49:1024-1027.

8. Goske MJ. Image gently: child-sizing radiation dose for children [comment]. JAMA Pediatr. 2013;167:1083.

9. Gelfand MJ, Parisi MT, Treves ST; Pediatric Nuclear Medicine Dose Reduction Workgroup. Pediatric radiopharmaceutical administered doses: 2010 North American consensus guidelines. J Nucl Med. 2011;52:318-322.

10. Fahey FH, Ziniel SI, Manion D, Treves ST. Effects of Image Gently and the North American guidelines: administered activities in children at 13 North American pediatric hospitals. J Nucl Med. 2015;56:962-967.

11. AHA Guide. Chicago, IL: American Hospital Association; 2010.

12. Title 10 of Code of Federal Regulations, part 35, section 35.3045: report and notification of a medical event. U.S. Nuclear Regulatory Commission website. http://www.nrc.gov/reading-rm/doc-collections/cfr/part035/part035-3045.html. Updated December 2, 2015. Accessed May 3, 2016.

13. Pediatric injected activity tool. SNMMI website. http://www.snmmi.org/ pedactivitytool. Updated April 16, 2014. Accessed May 3, 2016.

14. Dosage card. EANM website. http://www.eanm.org/publications/dosage_calculator. php?navId=285. Version 1.2.2014. Accessed May 3, 2016.

15. Fahey FH, Bom HH, Chiti A, et al. Standardization of administered activities in pediatric nuclear medicine part 2: a report of the first nuclear medicine global initiative. J Nucl Med. March 31, 2016 [Epub ahead of print].

16. Lassmann M, Treves ST; EANM/SNMMI Paediatric Dosage Harmonization Working Group. Paediatric radiopharmaceutical administration: harmonization of the 2007 EANM paediatric dosage card (version 1.5.2008) and the 2010 North American consensus guideline. Eur J Nucl Med Mol Imaging. 2014;41:1036-1041. 